\title{
On the dangers of conflating strong and weak versions of a theory of consciousness
}

Matthias Michel ${ }^{1}$, Hakwan Lau ${ }^{2,3,4,5}$

1. Sciences, Normes et Démocratie, Sorbonne Université, CNRS

2. Department of Psychology, UCLA

3. Brain Research Institute, UCLA

4. Department of Psychology, The University of Hong Kong

5. State Key Laboratory for Brain and Cognitive Sciences, The University of Hong Kong

Correspondence: matthias.michel.curtil@gmail.com; hakwan@gmail.com

\begin{abstract}
Some proponents of the Integrated Information Theory (IIT) of consciousness profess strong views on the Neural Correlates of Consciousness (NCC), namely that large swathes of the neocortex, the cerebellum, at least some sensory cortices, and the so-called limbic system are all not essential for any form of conscious experiences. We argue that this connection is not incidental. Conflation between strong and weak versions of the theory has led these researchers to adopt definitions of NCC that are inconsistent with their own previous definitions, inadvertently betraying the promises of an otherwise fruitful empirical endeavour.
\end{abstract}

\section{NCCs, Markers, and Constituents}

"We are not receptive to physicists trying to apply exotic physics to the brain, about which they seem to know very little, and even less about consciousness." - Crick \& Koch (2003)

Identifying the neural correlates of consciousness (NCC) has been a central research program in consciousness science for decades. In common neurobiological language, the key term would have been 'mechanisms' rather than 'correlates'. The term 'correlates' was so carefully chosen to remain neutral on conceptual issues regarding the exact metaphysical relation between consciousness and the NCCs (Crick \& Koch, 1990). It was recognized early on that such questions are difficult, and best set aside until we have clearer answers on the NCC.

In this article, we introduce a conceptual distinction between NCCs, markers of consciousness, and constituents of consciousness. We argue that a failure to distinguish between these different concepts is limiting the progress of the NCC project. We will illustrate this point with the case of the Integrated Information Theory (IIT) of consciousness. 
An NCC is "the minimal set of neuronal events and mechanisms jointly sufficient for a specific conscious percept" (Koch, 2004, p.16). That is, NCCs are the minimal neural difference makers that are jointly sufficient for a mental state to be conscious, rather than unconscious. This is just another way of saying that, all other things being equal, if a mental state is unconscious, activation of the NCC should be sufficient for making it conscious.

We distinguish the NCC from the constituents of consciousness. If they exist, constituents of consciousness are neural, or physical states that are identical with consciousness. This means that the constituents of consciousness should be sufficient - and necessary - for consciousness, just as $\mathrm{H} 2 \mathrm{O}$ is the constituent of water.

Research has focused on finding NCCs rather than constituents of consciousness because the necessity condition is generally considered too strong (Chalmers, 2000). Indeed, if a neural state is not only sufficient, but also absolutely necessary for a specific conscious experience to occur, it means that that experience is not multiply realizable (Chalmers, 2000; Michel, 2018; Morales \& Lau, forthcoming). That is, as in the case of water being identical to $\mathrm{H} 2 \mathrm{O}$, we cannot replace hydrogen or oxygen with something else to produce water. $\mathrm{H} 2 \mathrm{O}$ is the only possible recipe. This claim would, in effect, be contradictory with the widely acknowledged phenomenon of degeneracy in biology (Edelman \& Gally, 2001; Tononi et al., 1999) - which states that given a context, the same biological function can be carried out by different substrates, giving the impression of redundancy. If the same conscious experience could be realized with slightly different neuronal ensembles which may largely overlap but are not exactly identical, an NCC at this level cannot be considered strictly necessary for a particular experience. We will come back to this point below.

Lastly, we distinguish constituents and NCCs from the markers of consciousness. By markers we mean general evidence that can be used to determine whether subjects have conscious mental states or not. In that sense, NCCs can be used as markers of consciousness, because if a person is able to entertain a specific conscious experience, it demonstrates that the person is conscious in at least some limited sense. But not all markers of consciousness are NCCs, or constituents of consciousness. These markers may be very general, and might not specifically reflect the mechanisms directly responsible for consciousness.

To illustrate this distinction, let us consider the fact that when one is conscious rather than in a coma, one is more capable of producing behavior, thoughts, and memory. Therefore, a neural marker for sophisticated cognition may generally be used as a marker for consciousness. But such marker may not be the minimally sufficient condition for specific conscious experiences; one may need much less for a specific single experience to arise. And such marker may also not be necessary for conscious experiences; one might be able to entertain a specific experience without sophisticated cognition. Still, it could be that, in general, one can pragmatically use a neural marker for sophisticated cognition as a marker for consciousness, as consciousness and cognition might generally correlate, at least in humans. 
We will explain how a failure to carefully distinguish between NCCs, markers, and constituents of consciousness, is at the heart of a conflation between a strong version of the Integrated Information Theory of consciousness, and a weaker, empirical version.

\section{Empirical vs Fundamental IIT}

The recent 'rise' of IIT is somewhat paradoxical. On the one hand, the theory has been promoted with an unusual level of enthusiasm. For example, it has been claimed that IIT is "currently accepted as one of the most compelling explanations about what consciousness is" 1 (italics ours), that it is a "gigantic step in the final resolution of the ancient mind-body problem" (Koch, 2014), and even that it yields "a new kind of scientific spirituality"2.

On the other hand, arguments and criticisms against the theory abound (Barrett \& Mediano, 2019; Bayne, 2018; Cerullo, 2015; Doerig et al., 2019; Pautz, 2019). To answer one of those criticisms, proponents of IIT ended up acknowledging that, according to the theory, even a set of 'inactive' logic gates would be conscious (Aaronson 2014a,b; Tononi, 2014). Such possibilities have been openly denounced as "untestable" by many (Michel et al., 2019) ${ }^{3}$. Overall, the theory has not impressed active researchers as much as non-experts outside the field (Michel et al., 2018).

We suspect that this striking difference in opinions is due to a conflation between two versions of the theory, which, following Mediano et al. (2018), we call Empirical IIT, and Fundamental IIT. While the former may have some merits, we are unsure about the latter.

Empirical IIT is the view that some measures of complexity of brain networks can be used to detect states of consciousness, i.e., whether subjects have subjective experiences (such as during wakefulness, or dreams), or not (such as being in a coma, anesthetized, or dreamlesssleep). That is, Empirical IIT takes complexity to be a marker of consciousness.

On this view, the choice of an exact quantitative measure of complexity is an empirical matter. For instance, if it turns out that some measure of complexity does a better job at categorizing states of consciousness accurately, this would count as empirical data in favor of using that measure. Overall, despite some contradictory evidence (Noel et al., 2019, Sasai et al., 2016; Tajima et al., 2015) ${ }^{4}$, there is support for Empirical IIT (Barttfeld et al., 2015; Bodart et al., 2017; Casali et al., 2013, Casarotto et al., 2016; Demertzi et al., 2019; Ferrarelli et al., 2010;

\footnotetext{
${ }^{1}$ See: https://qz.com/709969/2300-years-later-platos-theory-of-consciousness-is-being-backed-up-byneuroscience/

${ }^{2}$ https://www.huffpost.com/entry/post_b_8160914

${ }^{3}$ This point was also made in a recent letter to the $\mathrm{NIH}$, by dozens of researchers in the field, available online here: https://tinyurl.com/y5wokv9g.

${ }^{4}$ See http://inconsciousnesswetrust.blogspot.com/2017/08/how-to-make-iit-and-other-theories-of.html for discussion as to why Sasai et al. (2016) and Tajima et al. (2014), and some other studies are considered potentially contradictory evidence against Empirical IIT.
} 
Rosanova et al., 2012; Ruiz de Miras et al., 2019; Sarasso et al., 2015; Tagliazucchi et al., 2013). We can consider Empirical IIT scientifically plausible.

On the other hand, Fundamental IIT is the view that a specific form of complexity (integrated information) is identical with consciousness (Oizumi et al., 2014). That is, Fundamental IIT posits that a specific form of complexity is the constituent of consciousness. Specific conscious experiences are also exactly identified with specific states of a network with this particular form of complexity (Tsuchiya et al., 2015; Tsuchiya, 2017).

On Fundamental IIT, the exact measure of complexity to adopt is not a matter to be determined by the data. Rather, it stems from some assumptions, or axioms, that are meant to be selfevidently true (Oizumi et al., 2014; Tononi \& Koch, 2015; Tononi et al., 2016). From there, one is supposed to mathematically derive the specific notion of complexity that is relevant for consciousness, namely, integrated information. We will argue that Fundamental IIT is not in line with current scientific knowledge and practice. It may also jeopardize the NCC project.

\section{Conflating general markers and constituents}

Let's assume that measures of complexity can be successfully used as markers of states of consciousness, as claimed by Empirical IIT. Importantly, we have to recognize that this would be entirely compatible with the rejection of Fundamental IIT.

That is to say, data in favor of Empirical IIT may be compatible with other theories, such as the Global Workspace Theory $(\mathrm{GWT})^{5}$. According to GWT, consciousness results from the global broadcast of information to a wide variety of neuro-cognitive modules through a "global workspace". If one supposes that this global broadcast is best achieved in a network of high complexity, GWT can account for currently available evidence in support of Empirical IIT, without invoking the controversial conjectures of Fundamental IIT.

Also, that some measures of complexity can be used as markers of states of consciousness does not directly support the claim that integrated information, which is a specific kind of complexity, is identical with consciousness. Because the exact calculation of the degree of integrated information in real biological systems is computationally challenging (Barrett \& Mediano, 2019) ${ }^{6}$, studies currently rely on proxy measures. Due to the approximate nature of the measurements, the relevant results cannot support claims concerning constituents, rather than markers. That is, they support Empirical, not Fundamental IIT.

\footnotetext{
${ }^{5}$ As apparently acknowledged by proponents of IIT (Koch says that "The global workspace theory and integrated information theories are not mutually exclusive", see: https://www.livescience.com/47096theories-seek-to-explain-consciousness.html)

${ }^{6}$ As remarked by Barrett \& Mediano (2019), this is because "the computation time required to compute [integrated information] grows faster than exponentially with the number of system components" (p.1).
} 
On this note, we remark there are many different approximated measures of integrated information, and they do not empirically converge (Mediano et al., 2018). Given the same data, indeed, Fundamental IIT can both be supported or falsified by these measures, depending on which approximation we choose to adopt. Relatedly, there is currently a debate as to whether Fundamental IIT is empirically falsifiable at all (Doerig et al., 2019).

Finally, and perhaps most importantly, in order to interpret the successful results of complexity measures as support for the claim that consciousness is identical with some specific kind of complexity, proponents of Fundamental IIT would have to rule out important confounds. For instance, a wide variety of cognitive capacities also differ between wakeful and unconscious subjects. As such, it could be that complexity measures reflect these differences, instead of differences in consciousness per se. As a result, to use data obtained with complexity measures of wakeful versus unconscious subjects to support Fundamental IIT, proponents of Fundamental IIT would have to provide good reasons for believing that complexity indexes consciousness, and not cognition, or any other factor that varies between wakeful and unconscious individuals.

To be clear, again, this latter argument applies to Fundamental IIT, but not to Empirical IIT. As long as complexity does correlate with differences in states of consciousness, whatever the reason behind this correlation, complexity measures can be used as markers of consciousness. It is an entirely different matter, however, to claim that complexity should be identified with consciousness, as do proponents of Fundamental IIT.

Therefore, if we do not confuse markers and constituents of consciousness, it should be clear that reasons for finding Empirical IIT appealing generally do not extend to Fundamental IIT.

\section{Conflating constituents and NCCs}

To make claims about the constituents of consciousness, we need to do more than just ruling out some confounds. Identity relationships like that between water and $\mathrm{H} 2 \mathrm{O}$ are very strong statements to make. To do so, we first need to have a very good understanding of the relevant substrates (hydrogen and oxygen in this case), their causal properties, how they interact, as well as to make sure that they are necessary. However, in the case of Fundamental IIT, it is unclear what kind of physical states are supposed to be identical to consciousness.

This is because, on Fundamental IIT, the measure of complexity to be assessed concerns some 'nodes' of a physical system, that are connected with each other. These nodes are either on, or off. They are either connected to other nodes or not. Of course, we already know that neurons

are much more complex than simple on/off nodes, as they show degrees of intensity of firing (rate), different dynamics in firing, different types of connections with each other (Kandel et al., 
2012). As such, proponents of Fundamental IIT acknowledge that these 'nodes' likely operate at a "different level". That is, they are not necessarily neurons, as one may intuitively think.

Despite the complete lack of direct empirical support for Fundamental IIT, at this yet-to-bespecifed, possibly sub-neuronal level, let us assume that some such substrate can be found to be correlated with consciousness. Even so, such correlation will only support that these constituents may be candidates for the NCC, but not that they are identical to conscious experiences. To make the latter claim, one would need to show that they are absolutely necessary, just like we cannot replace oxygen or hydrogen with something else if we want to produce water.

This kind of strong identity relationships are unlikely to be found in most areas of biology, and neuroscience in particular, in which we generally try to identify mechanisms (Craver, 2007). Indeed, if a given neural state $\mathrm{N}$ is necessary for a given conscious experience $\mathrm{C}, \mathrm{C}$ cannot obtain unless $\mathrm{N}$ does. This implies that $\mathrm{C}$ could not be realized by a very slightly different neural state, N2. Let's say that $N$ involves tens of thousands of 'nodes'. N2 would be considered different in this context even if only the connection between two out of these nodes were modified, with everything else being identical to $\mathrm{N}$. This would be broadly inconsistent with the phenomenon of biological degeneracy, which is particularly prominent in highly complex systems - a point with which proponents of IIT should be familiar (Edelman \& Gally, 2001; Tononi et al., 1999).

We therefore advocate maintaining the traditional definition of NCC. If we are to make such a radical modification of the definition, to move from mere correlation to absolute identity, we should do so explicitly. Unfortunately, we suspect this change in definition has recently slipped into current debates tacitly.

\section{$\underline{\text { NCC confused }}$}

One of the most striking claims made by proponents of IIT, beside panpsychism (Tononi, 2014), may be their views on the NCC. In particular, Christof Koch and colleagues have made the strong claim that most areas outside of a putative region in the 'posterior cortex' are not home to the NCC. The excluded regions presumably include the insula, amygdala, and different parts of the prefrontal cortex (PFC). In particular, this is meant to concern almost all conscious experiences, not just conscious perception (Koch et al., 2016; Tononi et al., 2016).

We remark that this claim may seem grossly incompatible with standard textbook knowledge, especially regarding conscious experiences of hunger, emotions, pain, intentions, thoughts, etc. But perhaps analyzing the case of the role of PFC for conscious visual perception is more

\footnotetext{
${ }^{7}$ Personal communications with Andrew Haun, and Masafumi Oizumi. See a conversation between Hakwan Lau, Masafumi Oizumi, and Richard Brown, available here: https://www.youtube.com/watch?v=8AP6YQrwaNO . Discussion of this point is roughly between $21 \mathrm{~m} 38 \mathrm{~s}$ $31 \mathrm{~m} 28 \mathrm{~s}$.
} 
illuminating still, because Koch himself used to hold exactly the opposite view. Here, we suggest that his more recent view on this matter is likely the result of a new commitment to the search for the constituents of consciousness, instead of the search for the NCCs.

Curiously, Koch's new justification for excluding PFC from the NCC is largely based on the very same data that previously led him to accept that the PFC was an NCC for conscious visual experiences. Indeed, Crick \& Koch (1998, p.103; see also Koch \& Crick, 2004) hypothesized that patients with bilateral lesions to the PFC may be able to respond unconsciously to visual stimuli, without being conscious of those stimuli. However, some well-known cases of bilateral lesions to the PFC had long been taken as indicating that patients with these lesions were not blind (Brickner, 1954). At that time, Koch concluded that these cases were not decisive for settling the debate on whether PFC was a neural correlate of consciousness (Crick \& Koch, 1998, p.103; Koch \& Crick, 2004).

To our minds, these cases remain as indecisive as they were two decades ago, in part because the completeness of these lesions remains a matter of dispute (Odegaard et al., 2018). Meanwhile, it is now known that even in unilateral PFC lesions, there are in fact specific perceptual deficits (Fleming et al., 2014).

But more importantly, in recent correspondence, Koch insists that the lack of complete abolishment of conscious perception in these unilateral cases is evidence for writing off the entire PFC. Specifically, according to his current thinking, disruption of any part of the NCC should "necessarily" cause changes in conscious experience ${ }^{8}$.

This 'new' definition of the NCC, involving necessity, is in direct contradiction with the original definition, as we explained in the opening section. For example, a group of neurons in the right PFC may be on their own minimally sufficient for a conscious experience to occur. But once lesioned, neurons in the left PFC may take over to perform the same function. This kind of dynamic reorganization of function in the PFC has also been empirically demonstrated (Voytek et al., 2010).

However, if one is committed to Fundamental IIT, specifically the claim of neural identity, one may well hold that degeneracy does not apply for consciousness. This seems to be the most charitable interpretation of the inconsistency between Koch's previous and current views on the NCC.

So, conflating the constituents and the NCCs probably has already had an impact on how current research and debates are conducted.

\footnotetext{
${ }^{8}$ In an email correspondence dated June 24, 2018, Koch wrote: "Any change in this NCC (via a different stimulus, or causal intervention such as TMS, optogenetics, drugs etc) will, of necessity, change the character of the experience (including having no experience). If the background conditions change but the NCC does not, the experience will likewise not change." (italics ours). Quoted with explicit permission.
} 


\section{Concluding remarks}

We have compared two versions of a theory of consciousness. While there is some empirical support for the weaker version (Empirical IIT), going from there to a much stronger version (Fundamental IIT) seems to require an unscientific leap of faith. The points we have made here are not specific to IIT per se. In general we should not make weighty claims beyond what is warranted by evidence. In analyzing the case of IIT, we also illustrate two culprits limiting progress in consciousness science.

The first mistake is to interpret evidence in favor of using a neural state as a marker of consciousness as evidence for the mechanistic relevance of this neural state for consciousness. This point applies specifically to the study of states of consciousness, in which we might often be too quick in identifying markers of consciousness with NCCs.

The second mistake is to confound NCCs with neural constituents of consciousness. As we have seen, doing so could lead to the rejection of the well-known biological phenomenon of degeneracy, and to controversial interpretations of lesion studies. We would do well not to let our theoretical commitments derail the NCC project.

\section{$\underline{\text { References }}$}

Aaronson, S. (2014a). Why I am not an integrated information theorist (or, the unconscious expander). www.scottaaronson.com/blog/?p=1799.

Aaronson, S. (2014b). Giulio Tononi and Me: A Phi-nal Exchange. https://www.scottaaronson.com/blog/?p=1823.

Barrett, A. B., \& Mediano, P. A. . (2019). The Phi Measure of Integrated Information is not WellDefined for General Physical Systems. Journal of Consciousness Studies, 26(1-2).

Barttfeld, P., Uhrig, L., Sitt, J. D., Sigman, M., \& Jarraya, B. (2015). Signature of consciousness in the dynamics of resting-state brain activity. Proceedings of the National Academy of Sciences, 112(37), E5219-E5220.

Bayne, T. (2018) On the axiomatic foundations of the integrated information theory of consciousness, Neuroscience of Consciousness, Volume 2018, Issue 1.

Bodart, O., Amico, E., Wannez, S., Gomez, F., Casarotto, S., Rosanova, M., ... Martens, G. (2017). Global structural and effective connectivity in patients with chronic disorders of consciousness. Brain Stimulation: Basic, Translational, and Clinical Research in Neuromodulation, 10(2), 353.

Boly, M., Massimini, M., Tsuchiya, N., Postle, B. R., Koch, C., \& Tononi, G. (2017). Are the Neural Correlates of Consciousness in the Front or in the Back of the Cerebral Cortex? Clinical and Neuroimaging Evidence. The Journal of Neuroscience, 37(40), 9603-9613. 
Brickner RM (1952) Brain of Patient A after bilateral frontal lobectomy: status of frontal-lobe problem. AMA Arch Neurol Psychiatry 68:293-313.

Casali, A. G., Gosseries, O., Rosanova, M., Boly, M., Sarasso, S., Casali, K. R., ... Massimini, M. (2013). A Theoretically Based Index of Consciousness Independent of Sensory Processing and Behavior. Science Translational Medicine, 5(198), 198ra105-198ra105.

Casarotto, S., Comanducci, A., Rosanova, M., Sarasso, S., Fecchio, M., Napolitani, M., ... Massimini, M. (2016). Stratification of unresponsive patients by an independently validated index of brain complexity. Annals of Neurology, 80(5), 718-729.

Chalmers, D. (2000). What is a Neural Correlate of Consciousness? In T. Metzinger (Ed.), Neural Correlates of Consciousness: Empirical and Conceptual Issues (pp. 1-33). MIT Press.

Craver, C. (2007). Explaining the Brain. Oxford University Press.

Crick, F., \& Koch, C. (1990). Towards a neurobiological theory of consciousness. Seminars in the Neurosciences, 2(263-275), 203.

Crick, F., \& Koch, C. (2003). A framework for consciousness. Nature Neuroscience, 6(2), 119126.

Cerullo, M. A. (2015). The Problem with Phi: A Critique of Integrated Information Theory. PLoS Computational Biology, 11(9), 1-12.

Demertzi, A., Tagliazucchi, E., Dehaene, S., Deco, G., Barttfeld, P., Raimondo, F., ... Sitt, J. D. (2019). Human consciousness is supported by dynamic complex patterns of brain signal coordination. Science Advances, 5(2), eaat7603.

Doerig, A., Schurger, A., Hess, K., \& Herzog, M. H. (2019). The unfolding argument: Why IIT and other causal structure theories cannot explain consciousness. Consciousness and Cognition, 72, 49-59.

Edelman, G. M., \& Gally, J. A. (2001). Degeneracy and complexity in biological systems. Proceedings of the National Academy of Sciences, 98(24), 13763-13768.

Ferrarelli, F., Massimini, M., Sarasso, S., Casali, A., Riedner, B. A., Angelini, G., ... Pearce, R. A. (2010). Breakdown in cortical effective connectivity during midazolam-induced loss of consciousness. Proceedings of the National Academy of Sciences, 107(6), 2681 LP - 2686.

Fleming, S. M., Ryu, J., Golfinos, J. G., \& Blackmon, K. E. (2014). Domain-specific impairment in metacognitive accuracy following anterior prefrontal lesions. Brain, 137(Pt 10), 2811-2822.

Kandel, E. R., Schwartz, J. H., Jessell, T. M., Siegelbaum, S. A., and Hudspeth, A. J. (2012). Principles of neural science. McGraw-Hill Education, 5th edition.

Koch, C. (2004). The Quest for Consciousness: A Neuroscientific Approach, Roberts \& Co.

Koch, C. \& Crick, F. (2004). The Neuronal Basis of Visual Consciousness. In: The visual neurosciences. MIT Press, Cambridge, Mass., pp. 1682-1694.

Koch, C. (2014). Is Consciousness Universal? Scientific American. January 1.

Koch, C., Massimini, M., Boly, M., \& Tononi, G. (2016). Neural correlates of consciousness: progress and problems. Nature Reviews Neuroscience, 17(5), 307-321. 
Mediano, P.A.M., Seth, A.K., \& Barrett, A.B. (2019). Measuring integrated information: Comparison of candidate measures in theory and simulation. Entropy, 21, 17.

Michel, M. (2018). Fish and Microchips: on fish pain and multiple realization. Philosophical Studies.

Michel, M., Fleming, S. M., Lau, H., Lee, A. L. F., Martinez-Conde, S., Passingham, R. E., ... Liu, K. (2018). An Informal Internet Survey on the Current State of Consciousness Science. Frontiers in Psychology, 9, 2134.

Michel, M., Beck, D., Block, N., Blumenfeld, H., Brown, R., Carmel, D., ... Yoshida, M. (2019). Opportunities and challenges for a maturing science of consciousness. Nature Human Behaviour, 3(2), 104-107.

Morales, J. \& Lau, H. (forthcoming). The neural correlates of consciousness. In Uriah Kriegel (ed.), The Oxford Handbook of the Philosophy of Consciousness. Oxford University Press.

Noel, J.-P., Ishizawa, Y., Patel, S. R., Eskandar, E. N., \& Wallace, M. T. (2019). Leveraging non-human primate multisensory neurons and circuits in assessing consciousness theory. BioRxiv, 584516.

Oizumi, M., Albantakis, L., \& Tononi, G. (2014). From the Phenomenology to the Mechanisms of Consciousness: Integrated Information Theory 3.0. PLoS Computational Biology, 10(5).

Pautz, A. (2019). What is the Integrated Information Theory of Consciousness?: A Catalogue of Questions. Journal of Consciousness Studies, 26(1-2).

Rosanova, M., Gosseries, O., Casarotto, S., Boly, M., Casali, A. G., Bruno, M.-A., ... Massimini, M. (2012). Recovery of cortical effective connectivity and recovery of consciousness in vegetative patients. Brain, 135(4), 1308-1320.

Ruiz de Miras, J., Soler, F., Iglesias-Parro, S., Ibáñez-Molina, A. J., Casali, A. G., Laureys, S., ... Langa, J. A. (2019). Fractal dimension analysis of states of consciousness and unconsciousness using transcranial magnetic stimulation. Computer Methods and Programs in Biomedicine, 175, 129-137.

Sarasso, S., Boly, M., Napolitani, M., Gosseries, O., Charland-Verville, V., Casarotto, S., ... Massimini, M. (2015). Consciousness and Complexity during Unresponsiveness Induced by Propofol, Xenon, and Ketamine. Current Biology, 25(23), 3099-3105.

Sasai, S., Boly, M., Mensen, A., \& Tononi, G. (2016). Functional split brain in a driving/listening paradigm. Proceedings of the National Academy of Sciences, 113(50), 14444 LP - 14449.

Tajima, S., Yanagawa, T., Fujii, N., Toyoizumi, T. (2015). Untangling Brain-Wide Dynamics in Consciousness by Cross-Embedding. PLoS Computational Biology, 11(11): e1004537.

Tagliazucchi, E., von Wegner, F., Morzelewski, A., Brodbeck, V., Jahnke, K., \& Laufs, H. (2013). Breakdown of long-range temporal dependence in default mode and attention networks during deep sleep. Proceedings of the National Academy of Sciences, 110(38), 15419 LP - 15424.

Tal, E. (2013). Old and New Problems in philosophy of measurement. Philosophy Compass, 12, $1159-1173$. 
Tononi, G., Sporns, O., \& Edelman, G. M. (1999). Measures of degeneracy and redundancy in biological networks. Proceedings of the National Academy of Sciences, 96(6), 3257 LP - 3262.

Tononi, G. (2014). Why Scott should stare at a blank wall and reconsider (or, the conscious grid). https://www.scottaaronson.com/blog/?p=1823.

Tononi, G., \& Koch, C. (2015). Consciousness: Here, There, and Everywhere? Philosophical Transactions of the Royal Society of London. Series B, Biological Sciences, 31(1), 12-19.

Tononi, G., Boly, M., Massimini, M., \& Koch, C. (2016). Integrated information theory: from consciousness to its physical substrate. Nature Reviews Neuroscience, 17(7), 450-461.

Tsuchiya, N., Taguchi, S., \& Saigo, H. (2015). Using category theory to assess the relationship between consciousness and integrated information theory. Neuroscience Research, 107, 1-7.

Tsuchiya, N. (2017). "What is it like to be a bat? "- a pathway to the answer from the integrated information theory. Philosophy Compass, 12, 1-13.

Voytek, V., Davis, M., Yago, E., Barcelo, F., Vogel, E. K., Knight, R.T. (2010). Dynamic neuroplasticity after Human prefrontal cortex damage. Neuron, 68:401- 408. 